\title{
Gene therapy regulation: could in-body editing fall through the net?
}

\author{
Miranda Mourby ${ }^{1} \cdot$ Michael Morrison ${ }^{1}$
}

Received: 12 December 2019 / Revised: 18 February 2020 / Accepted: 3 March 2020 / Published online: 25 March 2020

(c) European Society of Human Genetics 2020

\begin{abstract}
Somatic gene therapies may be authorised for marketing in the EU under the advanced therapy medicinal product regulation. These therapeutic compounds are sufficiently novel and complex in their potential effects to require specialist evaluation. However, the current definition of gene therapy medicinal products ('GTMP') risks excluding molecules which are not manufactured through techniques involving recombination. We consider the way, in which the 'recombinant nucleic acid' aspect of the GTMP definition is challenged by developments in gene-editing technology, and why a broader scope of GTMP regulation may be desirable.
\end{abstract}

\section{Introduction}

While human germline gene-editing is prohibited under the EU clinical trials regulation [1], somatic (apparently nonheritable) modification techniques have already received marketing authorisation from the European Commission. Cornel et al. consider how somatic gene-editing will be evaluated as gene therapy medicinal products ('GTMPs') under the advanced therapy medicinal products ('ATMP') regulation [2], concluding that this pathway will be difficult for novel therapies to navigate [3]. An additional, important consideration is whether gene-editing molecules will qualify as GTMPs in the first place.

In-body gene therapy has been a clinical reality since the FDA approved Luxturna in 2017 [4]. The European Medicines Agency ('EMA'), which coordinates scientific evaluation of new therapies on behalf of the Commission, has published a number of favourable recommendations of products, which alter genetic material within the human body [5]. The difficulty with this trend, however, is that some in vivo products may not fall within the definition of a GTMP. A GTMP is defined as containing, or consisting of,

Supplementary information The online version of this article (https:// doi.org/10.1038/s41431-020-0607-y) contains supplementary material, which is available to authorised users.

Miranda Mourby

miranda.mourby@law.ox.ac.uk

1 Centre for Health, Law and Emerging Technologies ('HeLEX'), Faculty of Law, University of Oxford, Oxford, UK 'recombinant nucleic acid', but it is far from clear that nucleic acids within CRISPR will always be produced by recombination, and protein-based molecules such as zinc finger nucleases or TALENS could be out of scope altogether if they do not include any nucleic acid. If therapeutic gene-editing compounds fall outside the definition of a GTMP, they would have to be reviewed outside of the specialised scrutiny of the ATMP regulation.

\section{Methods and materials}

See Supplementary material, Box 3.

\section{Results and discussion}

The definition of a GTMP which can be aggregated from the ATMP regulation and the Medicinal Products Directive is:

(1) A biological medicinal product, i.e. its active substance must be a 'biological substance'. A biological substance is defined as one produced by or extracted from a biological source [6];

(2) This active substance must also contain or consist of a recombinant nucleic acid used in or administered to human beings with a view to regulating, repairing, replacing, adding or deleting a genetic sequence, and

(3) It must have a therapeutic, prophylactic or diagnostic effect, which relates directly to the recombinant nucleic acid sequence it contains, or to the product of genetic expression of this sequence [7]. 
To stretch the term 'recombinant nucleic acid,' as used in this definition, to cover all in vivo gene-editing is problematic, as it is by no means inevitable that the active nucleic acid will be produced through the 'recombination,' and some gene-editing molecules and vectors will not contain nucleic acid at all.

For example, CRISPR could be delivered as a ribonucleoprotein-i.e. a recombinant protein and synthetic guide ribonucleic acid. This RNA could be produced via in vitro transcription [8], a 'template'-based synthesis that would not fit the description of recombination in EU law, i.e.:

'techniques involving the formation of new combinations of genetic material by the insertion of nucleic acid molecules produced by whatever means outside an organism into any virus, bacterial plasmid or other vector system and their incorporation into a host organism in which they do not naturally occur but in which they are capable of continued propagation [9].'

The above definition is taken from the Directive governing Genetically Modified Organisms. The European Commission has acknowledged that some advanced therapies may contain or consist of GMO's [10], and the provisions of this Directive would also apply to any such products released or placed on the market, meaning its definitions are relevant for ATMP's.

While any form of synthesis would form 'new combinations of genetic material,' it is the definition's requirement for this formation to be via 'the insertion of nucleic acid molecules produced by whatever means outside an organism' which sets the legal definition of recombination apart from nucleic acid synthesis in general. This is a distinction recognised in the US National Institute for Health ('NIH') guidelines:

'recombinant and synthetic nucleic acids are defined as:

i. molecules that (a) are constructed by joining nucleic acid molecules and (b) that can replicate in a living cell, i.e., recombinant nucleic acids;

ii. nucleic acid molecules that are chemically or by other means synthesised or amplified, including those that are chemically or otherwise modified but can base pair with naturally occurring nucleic acid molecules, i.e., synthetic nucleic acids [11],

The NIH's definition reflects the conventional use of the word 'recombinant' to mean recombined following the insertion of, or joining together with, another nucleic acid [12]. It also reflects the CJEU's treatment of CRISPR-style modification as 'mutagenesis' in their Confederation Paysanne [13] judgement:
The Court of Justice of the European Union dealt with a request for a ruling as to whether organisms manipulated by 'new methods of mutagenesis' (the term used to refer collectively to gene-editing compounds such as CRISPR) qualified as Genetically Modified Organisms ('GMO's).

The Court reviewed the GMO Directive's list of qualifying techniques, which includes recombination. They noted (at paragraph 34 of their judgment) that this list did not include new methods or techniques of mutagenesis. Nevertheless, they held that the GMO Directive could apply as this list was not exhaustive.

By confirming that new methods of mutagenesis were not included in this list, even though recombinant nucleic acid techniques are listed therein, the Court drew an implicit distinction between the two.

The Confederation Paysanne judgement thus places gene-editing compounds in the category of mutagenesis, as opposed to recombinant nucleic acid techniques that make up the older generation of modifications, sometimes referred to as 'transgenesis' instead [14]. The distinction is important: if genes are therapeutically altered by anything other than a nucleic acid produced by recombination, the product in question should not fall within the definition of a GTMP. As in vivo gene-editing molecules are not cells, they could not be treated as somatic cell or tissue engineered therapies, and so consequently would fall out of scope of the ATMP regulation altogether, and thus outside of the particular scrutiny reserved for Advanced Therapy Medicinal Products. It is possible that the 'recombinant' criterion is not always applied in practice. Box 2 provides an example of a product designated a GTMP within a scientific recommendation even though it is not clear whether recombination was involved (see Supplementary material).

Regardless of the logic in this instance, it is foreseeable that active nucleic acid in a gene-editing molecule may not be formed through recombination, or delivered by a recombinant vector. Stretching the law to make the definition fit in such cases would only create confusion, uncertainty and inconsistency with the GMO Directive.

Furthermore, protein-based molecules, zinc finger nucleases and TALENs would not contain nucleic acids (recombinant or otherwise), and could, in theory, be delivered using a non-viral vector such as lipid nanoparticles [5], in which case neither the molecule nor the vector could be said to be producing a therapeutic effect directly related to a recombinant nucleic acid, and the compound would clearly fall outside the GTMP definition. 
The ATMP procedure is intended to be compulsory for advanced therapy medicinal products in order to 'overcome the scarcity of expertise in the Community, ensure a high level of scientific evaluation of these medicinal products [15]'. The narrow definition of GTMPs currently embodied in the ATMP regulation, as inherited from the Medicinal Products Directive, does little to ensure coverage of all compounds, which may be used for in-body human gene-editing. The NIH's definition could provide a starting point for broadening the gateway into GTMP regulation, so these new therapies are not left to patchwork-regulation at Member State level.

\section{Conclusion}

The definition of a GTMP should be as future-proof and technology neutral as possible. By specifying how a GTMP should be produced, the ATMP regulation risks excluding from consideration molecules produced by other means but which carry out similar functions, and carry therapeutic risks which require the same level of expert consideration. While it has been part of the Commission's action plan on ATMPs to reflect on emerging technologies in gene-editing [16], it is not clear whether the GTMP definition will be broadened to include in vivo gene-editing. The US National Academy of Sciences has recommended the use of existing gene therapy review mechanisms for somatic gene editing [17]; it would be helpful to clarify whether this should also be the case within the EU.

Acknowledgements The authors are grateful for the review and comments from Alex Faulkner, Andrew Webster, Ben Davies, Dianne Nicol, Edison Bicudo, Jane Kaye, Lisa Eckstein, and Phoebe Li. Any errors are the authors' own.

Funding The authors acknowledge support from the Leverhulme Trust project grant RPG-2017-330, Economic and Social Research Council grant number ES/P002943/1 (Biomodifying technologies and experimental space: Organisational \& Regulatory Implications for the Translation \& Valuation of Health Research).

\section{Compliance with ethical standards}

Conflict of interest The authors declare that they have no conflict of interest.

Publisher's note Springer Nature remains neutral with regard to jurisdictional claims in published maps and institutional affiliations.

\section{References}

1. Council Regulation (EU) 536/2014 of 16 April 2014 on clinical trials on medicinal products for human use, and repealing Directive 2001/20/EC [2014] OJ L 158/1, Article 90. This reflects the prohibition in Article 13 Oviedo Convention, to which some EU Member States are party.
2. Council Regulation (EC) 1394/2007 of 13 November 2007 on advanced therapy medicinal products and amending Directive 2001/ 83/EC and Regulation (EC) No 726/2004 [2007] OJ L324/121.

3. Cornel MC, Howard HC, Lim D, Bonham VL, Wartiovaara K. Moving towards a cure in genetics: what is needed to bring somatic gene therapy to the clinic? Eur J Hum Genet. 2019;27:484-7. https://doi.org/10.1038/s41431-018-0309-x. Accessed 21 Feb 2020.

4. Bender C. Regulating the gene-therapy revolution. Nature. 2018;564:S20-S22. https://doi.org/10.1038/d41586-018-07641-1. Accessed 21 Feb 2020

5. European Medicines Agency, 'Scientific recommendation on classification of advanced therapy medicinal products EMA/ 814824/2018', 21 November 2018. https://www.ema.europa.eu/ en/documents/report/scientific-recommendation-classification-a dvanced-therapy-medicinal-products-codonoptimized-mrna_en. pdf. Accessed 17 March 2020.

6. Council Directive 2001/83/EC of 6 November 2001 on the Community code relating to medicinal products for human use [2001] OJ L311/67, Annex I, Part I, paragraph 3.2.1.1 (b).

7. Ibid, Annex I, Part IV, paragraph 2.1.

8. Hempsted A. 'CRISPR 101: Ribonucleoprotein (RNP) delivery', addgene blog 6 September 2018 https://blog.addgene.org/crispr101-ribonucleoprotein-rnp-delivery. Accessed 21 Feb 2020.

9. Council Directive 2001/18/EC of 12 March 2001 on the deliberate release into the environment of genetically modified organisms and repealing. Council Directive 90/220/EEC [2001] OJ L 106/1, Annex 1A, Part 1, para (1).

10. European Commission, 'The Rules Governing Medicinal Products in the European Union Vol 4: Guidelines on good manufacturing practice specific to advanced therapy medicinal products' 22 November 2017, p. 80. https://ec.europa.eu/health/sites/health/ files/files/eudralex/vol-4/2017_11_22_guidelines_gmp_for_a tmps.pdf. Accessed 25 Feb 2020.

11. Department of Health and Human Services, National Institutes of Health. NIH guidelines for research involving recombinant or synthetic nucleic acid molecules, 2016. https://osp.od.nih.gov/wp-content/ uploads/2016/05/NIH_Guidelines.pdf. Accessed 21 Feb 2020.

12. Stephenson FH. 'Recombinant DNA' in calculations for molecular biology and biotechnology. Elsevier (2016). https://www. sciencedirect.com/book/9780128022115/calculations-for-molecula r-biology-and-biotechnology\#book-info. Accessed 17 March 2020.

13. Case C-528/16 Confédération paysanne \& ors v Premier ministre, Ministre de l'Agriculture, de l'Agroalimentaire et de la Forêt (25 July 2018), http://curia.europa.eu/juris/document/document.jsf; jsessionid=9ea7d0f130da2186623a9dfe47d78dfe9e06ce39e17e. e34KaxiLc3eQc40LaxqMbN4Pb3qTe0?text $=\&$ docid $=$ 204387 \&pageIndex $=0 \&$ doclang $=\mathrm{EN} \&$ mode $=$ req $\& \operatorname{dir}=\& o c c=$ first\&part=1\&cid=486410. Accessed 17 March 2020.

14. Garnett K. Hold your pipettes: The European Court of Justice's findings in Confédération Paysanne \& Others stirs GMOtions. RECEIL. 2019;28:349-55. https://doi.org/10.1111/reel.12291. Accessed 21 Feb 2020.

15. Council Regulation (EC) 1394/2007 of 13 November 2007 on advanced therapy medicinal products and amending Directive 2001/83/EC and Regulation (EC) No 726/2004 [2007] OJ L324/ 121, Recital 9.

16. European Commission \& European Medicines Agency, 'European Commission-DG Health and Food Safety and European Medicines Agency Action Plan on ATMPs', 2018. https://www. ema.europa.eu/en/documents/other/european-commission-dg-hea lth-food-safety-european-medicines-agency-action-plan-adva nced-therapy_en.pdf. Accessed 21 Feb 2020.

17. Coller B. Ethics of human genome editing. Annu Rev Med. 2019;70:289-305. https://doi.org/10.1146/annurev-med-112717094629. Accessed 21 Feb 2020. 